\title{
RESEARCH IN APPLICATION OF GEODETIC GPS RECEIVERS IN TIME SYNCHRONIZATION
}

\author{
Qinglan Zhang, Peng Zhang, Zhanyi Sun, Fan Wang, Xiaoqing Wang \\ National Geomatics Center of China - (qlzhang, zhangpeng, szy, wangfan)@ngcc.cn
}

\begin{abstract}
KEY WORDS: Time Synchronization, GPS Common-View, GPS
\end{abstract}
ABSTRACT: In recent years, with the development of satellite orbit and clock parameters accurately determining technology and the popularity of geodetic GPS receivers, Common-View (CV) which proposed in 1980 by Allan has gained widespread application and achieved higher accuracy time synchronization results. GPS Common View (GPS CV) is the technology that based on multi-channel geodetic GPS receivers located in different place and under the same common-view schedule to receiving same GPS satellite signal at the same time, and then calculating the time difference between respective local receiver time and GPST by weighted theory, we will obtain the difference between above local time of receivers that installed in different station with external atomic clock。

Multi-channel geodetic GPS receivers have significant advantages such as higher stability、 higher accuracy and more common-view satellites in long baseline time synchronization application over the single-channel geodetic GPS receivers. At present, receiver hardware delay and surrounding environment influence are main error factors that affect the accuracy of GPS common-view result. But most error factors will be suppressed by observation data smoothing and using of observation data from different satellites in multi-channel geodetic GPS receiver. After the SA (Selective Availability) cancellation, Using a combination of precise satellite ephemeris ,ionospheric-free dual-frequency P-code observations and accurately measuring of receiver hardware delay, we can achieve time synchronization result on the order of nanoseconds (ns).

In this paper, 6 days observation data of two IGS core stations with external atomic clock (PTB, USNO distance of two stations about $6000 \mathrm{~km}$ ) were used to verify the GPS common-view theory. Through GPS observation data analysis, there are at least 2- 4 common-view satellites and 5 satellites in a few tracking periods between two stations when the elevation angle is $15^{\circ}$, even there will be at least 2 common-view satellites for each tracking period when the elevation angle is $30^{\circ}$. Data processing used precise GPS satellite ephemeris, double-frequency P-code combination observations without ionosphere effects and the correction of the Black troposphere Delay Model. the weighted average of all common-viewed GPS satellites in the same tracking period is taken by weighting the root-mean-square error of each satellite, finally a time comparison data between two stations is obtained, and then the time synchronization result between the two stations (PTB and USNO) is obtained. It can be seen from the analysis of time synchronization result that the root mean square error of REFSV (the difference between the local frequency standard at the mid-point of the actual tracking length and the tracked satellite time in unit of $0.1 \mathrm{~ns}$ ) shows a linear change within one day, However the jump occurs when jumping over the day which is mainly caused by satellites position being changed due to the interpolation of two-day precise satellite ephemeris across the day. the overall trend of time synchronization result is declining and tends to be stable within a week-long time. We compared the time synchronization results (without considering the hardware delay correction) with those published by the International Bureau of Weights and Measures (BIPM), and the comparing result from a week earlier shows that the trend is same but there is a systematic bias which was mainly caused by hardware delays of geodetic GPS receiver. Regardless of the hardware delay, the comparing result is about between 102ns and 106ns. the vast majority of the difference within $2 \mathrm{~ns}$ but the difference of individual moment does not exceed $4 \mathrm{~ns}$ when taking into account the systemic bias which mainly caused by hardware delay. Therefore, it is feasible to use the geodetic GPS receiver to achieve the time synchronization result in nanosecond order between two stations which separated by thousands kilometers, and multi-channel geodetic GPS receivers have obvious advantages over single-channel geodetic GPS receivers in the number of common-viewing satellites. In order to obtain higher precision (e.g sub-nanosecond order) time synchronization results, we shall take account into carrier phase observations, hardware delay , and more error-influencing factors should be considered such as troposphere delay correction, multipath effects, and hardware delays changes due to temperature changes.

\section{INTRODUCTION}

As an important basic physical quantity, Frequency plays an important role in the study of national economy, defense construction and basic science. At present, the standard time used by all the countries or regions must be traced directly or indirectly to the unified time UTC which established by the International Bureau of Weights and Measures (BIPM). The main traceability way is GPS (Global Positioning System) common view method to comparing the time among laboratories which distance is very long ${ }^{[6-9]}$.

\section{BACKGROUND OF GPS COMMON VIEW}

ALLAN put forward the principle of GPS common view for the first time at the International Frequency Control Conference in 1980. In 1985, the method of GPS common view (GPS CV) was widely used to comparing the long-distance time and participating in TAI calculations. In 1994, ALLAN published a "GPS Timing Receiver Software Standardization Technical Guide" on MetroLogia which unifies the procedure of CCTV receiver software and the format of the document to further improving the accuracy of the common viewing ratio on behalf of the Group on GPS Time Transfer Standards (GGTTS).In the early 1990s, the GPS CV was widely be used ${ }^{[1]}$., GPS CV can be used to carrying out time synchronization among low-orbit satellites with the rapidly development of low-orbit satellites and the continuously improving accuracy of orbit determination $^{[10]}$.

In recent year, dual-frequency $\mathrm{P}$-code combined observations of geodesic GPS C/A multi-channel measurement receivers have been used for time sharing. Multi-channel co-viewing is 4 minutes ahead of the previous day, so that it can be compatible with 
single-channel co-viewing (ie, single-channel data conforming to the common view can be extracted from multi-channel data). The multi-channel common vision not only makes the common viewing ratio continuous, but also increases the amount of observation data, improving the accuracy and stability of the comparison.

\section{PRINCIPLE OF GPS COMMON VIEW}

3.1 observation equation of GPS C/A observations we can assume that station A achieved the satellite $\mathrm{j}$ at the moment $\mathrm{t}$ and obtained the pseudo-range measurement $\tilde{\rho}_{\mathrm{A}}^{j}(t)$., so the equation of pseudo-range observation which considering the influences is

$$
\tilde{\rho}_{\mathrm{A}}^{j}(t)=\rho_{A}^{j}(t)+c \sigma(t)_{A}^{j}+\Delta_{i}^{j}, I_{g}(t)+\Delta_{i}^{j}, T(t)+\sigma_{A}^{j}
$$

where $\tilde{\rho}_{\mathrm{A}}^{j}(t)$ is the pseudorange of P code, this is a P code dual-frequency combined observation with no ionosphere effect.

$\rho_{\mathrm{A}}^{\mathrm{j}}(t)$ is the geometric distance from satellite $\mathrm{j}$ to station A at epoch t.

$\sigma_{\mathrm{A}}^{\mathrm{j}}(t)$ is the clock difference between the receiver clock and the satellite clock at epoch $\mathrm{t}$.

$\Delta_{i}^{j}, I_{g}(t)$ is the influence of the ionosphere on the code pseudorange at epoch t. Due to the use of dual-frequency combined observations of the $\mathrm{P}$ code with no ionospheric effects, this effect can basically not be considered.

$\Delta_{i}^{j}, T(t)$ - the effect of the troposphere on the pseudorange at the epoch $\mathrm{t}$.

$$
\sigma_{A}^{j} \text { - Other influence items. }{ }^{[1]}
$$

\section{2 principle of GPS common view}

The method of GPS common view is refering to the time comparison between of the atomic clocks which arranged in any two locations in the perspective of the same GPS satellite using the same satellite's time signal received at the same time.

Let the clock time of station $\mathrm{A}$ is $\mathrm{t}_{\mathrm{A}}$; the clock time of station $\mathrm{B}$ is $\mathrm{t}_{\mathrm{B}}$; GPS time is $\mathrm{t}_{\mathrm{GPS}}$; The block diagram of basic principle of the common view is shown in Figure 1:

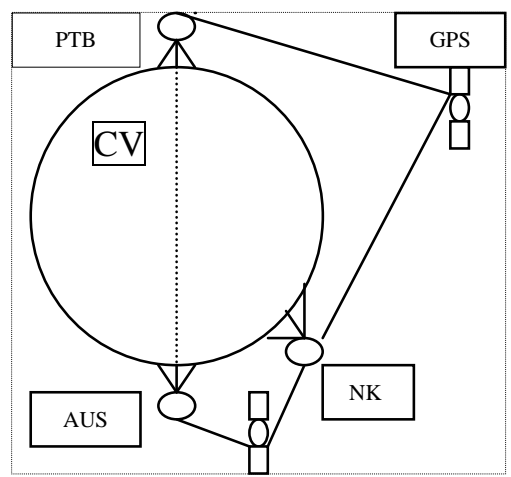

Figure 2: GPS common view

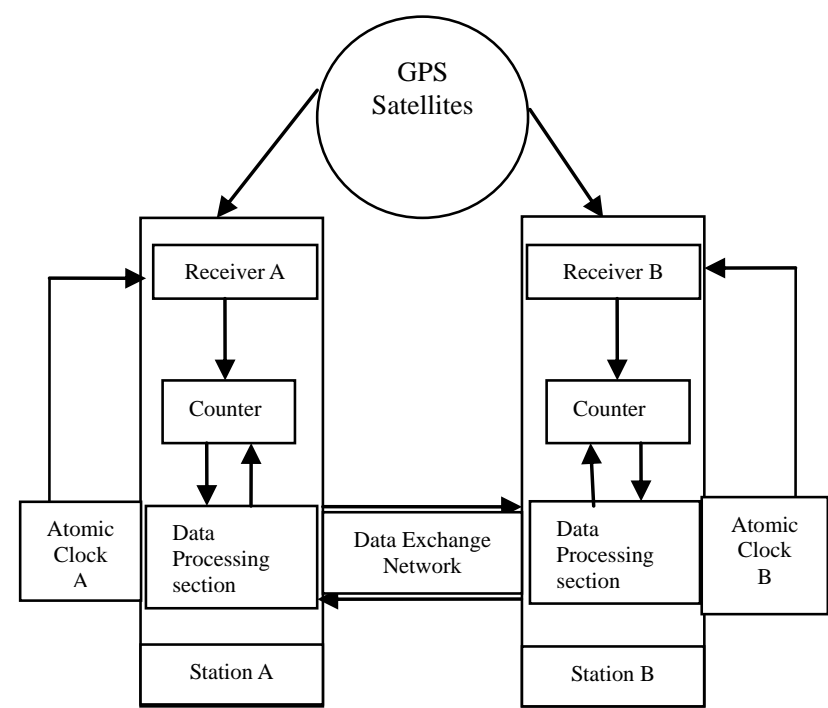

Figure 1: The principle of GPS common view

The basic principle of GPS common view (no delay error correction) used between stations A and B as follows: The GPS receivers arranged in the two different stations received the same one GPS satellite's signal at the same time under the same common timetable. The receiver's output represents The frequency pulse of the GPS time is sent to the time interval counter and compared with another frequency pulse' output to obtain the difference $\Delta t_{A G P S}$ between the local atomic clock time $t_{A}$ and the GPS time ${ }^{t_{G P S}}$. At the same time, the difference $\Delta t_{B G P S}$ between the local atomic clock time $\boldsymbol{t}_{B}$ and the GPS time $t_{G P S}$ is obtained. The difference of station $\mathrm{B}$ can be transmitted to the computers in station A by the communication network. Then we can obtain the difference between station A and station $\mathrm{B}$.

$$
\begin{aligned}
& \Delta t_{A G P S}=t_{A}-t_{G P S} \\
& \Delta t_{B G P \bar{S}}=t_{B}-t_{G P S}
\end{aligned}
$$

$\Delta t_{A G P S}-\Delta t_{B G P S}=t_{A}-t_{G P S}-\left(t_{B}-t_{G P S}\right)=t_{A}-t_{B}=\Delta t_{A B}$

After a series of results with multiple measurements, we can also calculate the average relative frequency deviation over a period of time. $f_{A}, f_{B}$ is two clock frequencies respectively, $\tau$ is the average time interval. The following formula shows:

$$
\frac{f_{A}-f_{B}}{f}=\frac{\Delta f}{f}=\frac{\Delta t_{A G P S}-\Delta t_{B G P S}}{\tau}
$$

Global three time laboratories use GPS common vision method for time transfer diagram:

\section{GGTTS GPS DATA FORMAT}

4.1 GGTTS data format introduction ${ }^{[10]}$

GGTTS GPS DATA FORMAT prepared by BIPM, including PRN, CL, MJD, STTIME, TRKL, ELV, AZTH, REFSV, SRSV, REFGPS, SRGPS, DSG, IOE, MDTR, SMDI MDIO, SMDI, MSIO, SMSI, ISG, FR, HC, FRC, CK.. At present, all international time laboratories using this standard format for time comparison, so that research institution conducts comparisons of atomic time scales conveniently in different regions. The sample of GGTTS file content is shown in Figure 3: 


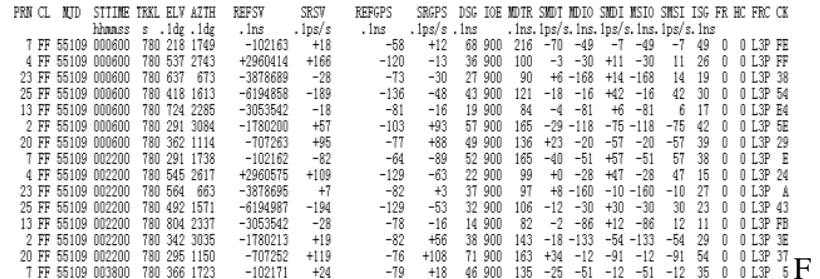

igure 3: Standard GGTTS Format Data File

The following will briefly describes the significance of the main parameters ${ }^{[11-12]}$ :

PRN: Number of the satellite, the available range is 1-32

MJD: Start date of Tracking satellite (reference UTC), 5-digit representation of reduced Julian day.

STTIME: The starting time of each tracking period, refers to UTC hours, minutes, and seconds.

TRKL: Actual tracking duration in seconds (s).

ELV: The vertical angle of the tracked satellite, in units of $0.1 \mathrm{dg}$.

AZTH: azimuth angle of the tracked satellite corresponding to the midpoint of the length. The unit is $0.1 \mathrm{dg}$.

REFSV: The difference between the local second pulse at the mid-point of the actual tracking length and the tracked satellite time, in units of $0.1 \mathrm{~ns}$.

REFGPS: The difference between local clock time and GPS time at the midpoint of the actual tracking length, in units of $0.1 \mathrm{~ns}$.

DSG: The root mean squared deviation of the difference between the actual value of REFGPS and the value on the fitted line.

\subsection{Least Squares Fitting Algorithm}

The technical guideline of the standard data format of the GPS common view developed by BIPM stipulates that the time for tracking satellites in each tracking period is 780 seconds (sampling rate is $1 \mathrm{~s}$ ), and every 15 seconds is a period of time and is divided into 52 segments. For each 15 seconds of the measured REFSV, REFGPS data using the least square method for quadratic curve fitting, respectively, to obtain 52 curve midpoint values. And then we use the least squares method to linearly fit the 52 midpoint values Respectively. The midpoint values of the straight line are REFSV and REFGPS, and the corresponding slopes are SRSV and SRGPS. ${ }^{[5]}$ data be:

Let the data sequence composed of time and calculation

$$
\left(x_{i}, y_{i}\right), i=1,2 \ldots 15
$$

We used a quadratic polynomial to fit this set of data sequences:

$$
q(x)=a_{0}+a_{1} x+a_{2} x^{2}
$$

Then you can get the sum of squared residuals:

$$
\mathrm{Q}\left(a_{0}, a_{1}, a_{2}\right)=\sum_{i=1}^{15}\left(q\left(x_{i}\right)-y_{i}\right)^{2}=\sum_{i=1}^{15}\left(a_{0}+a_{1} x_{i}+a_{2} x_{i}^{2}-y_{i}\right)^{2}
$$

According to the principle of multivariate function extremum , $\mathrm{Q}\left(a_{0}, a_{1}, a_{2}\right)$ partial derivatives of the parameters to be evaluated are zeroed and column cubed:

$$
\left(\begin{array}{l}
\frac{\partial Q}{\partial a_{0}} \\
\frac{\partial Q}{\partial a_{1}} \\
\frac{\partial Q}{\partial a_{2}}
\end{array}\right)=\left(\begin{array}{ccc}
\sum_{i=1}^{15} 1 & \sum_{i=1}^{15} x_{i} & \sum_{i=1}^{15} x_{i}^{2} \\
\sum_{i=1}^{15} x_{i} & \sum_{i=1}^{15} x_{i}^{2} & \sum_{i=1}^{15} x_{i}^{3} \\
\sum_{i=1}^{15} x_{i}^{2} & \sum_{i=1}^{15} x_{i}^{3} & \sum_{i=1}^{15} x_{i}^{4}
\end{array}\right)\left(\begin{array}{c}
a_{0} \\
a_{1} \\
a_{2}
\end{array}\right)-\left(\begin{array}{c}
\sum_{i=1}^{15} y_{i} \\
\sum_{i=1}^{i=1} x_{i} y_{i} \\
\sum_{i=1}^{15} x_{i}^{2} y_{i}
\end{array}\right)=\left(\begin{array}{l}
0 \\
0 \\
0
\end{array}\right)
$$

After obtaining the quadratic polynomial coefficients and then calculating the 52 midpoint values, the least squares linear fitting is performed on the 52 sets of data to obtain the fitted midpoint values REFSV, REFGPS, slopes SRSV, SRGPS, and Root deviation DSG. The linear fit is as follows: data is

Let the data sequence composed of time and calculation

$$
\left(x_{i}, y_{i}\right), i=1,2 \ldots .52 \text {, }
$$

he fitting line is:

$$
q(x)=a+b x
$$

Then you can get the sum of squared residuals:

$$
\mathrm{Q}(a, b)=\sum_{i=1}^{52}\left(q\left(x_{i}\right)-y_{i}\right)^{2}=\sum_{i=1}^{52}\left(a+b x_{i}-y_{i}\right)^{2}
$$

According to the principle of multivariate function extremum. The $\mathrm{Q}(a, b)$ derivative of the parameter to be evaluated is 0 , the columnar cubic formula:

$\left(\begin{array}{l}\frac{\partial Q}{\partial a} \\ \frac{\partial Q}{\partial b}\end{array}\right)=\left(\begin{array}{ll}\sum_{i=1}^{52} 1 & \sum_{i=1}^{52} x_{i} \\ \sum_{i=1}^{52} x_{i} & \sum_{i=1}^{52} x_{i}^{2}\end{array}\right)\left(\begin{array}{l}a \\ b\end{array}\right)-\left(\begin{array}{c}\sum_{i=1}^{15} y_{i} \\ \sum_{i=1}^{52} x_{i} y_{i}\end{array}\right)=\left(\begin{array}{l}0 \\ 0\end{array}\right)$

We can obtain the midpoint value REFSV, REFGPS, and the slope SRSV, SRGPS, and root mean square deviation DSG by finding the fitted line.

\section{EXAMPLES AND RESULTS ANALYSIS}

We calculated the GPS observations of the PTB and USNO two stations from 2009-10-5 to 2009-10-10 for 6 days respectively. The data was calculated using precise satellite ephemeris and non-ionosphere-influenced dual-frequency $\mathrm{P}$ code combined measurements. PTB is located in Braunschweig, Germany. The receiver of this station has an external frequency standard: cesium atomic clock with frequency of $20 \mathrm{MHz}$; USNO (US Naval Observatory Station,)located in Washington, DC, USA. The station also has an external frequency standard: hydrogen atomic clock and frequency is $5 \mathrm{MHz}$. The distance between the two stations is approximately $6000 \mathrm{~km}$, which is an extremely long baseline. Figure 4 shows the number of GPS satellites that can be viewed by two stations during each tracking period of the dav

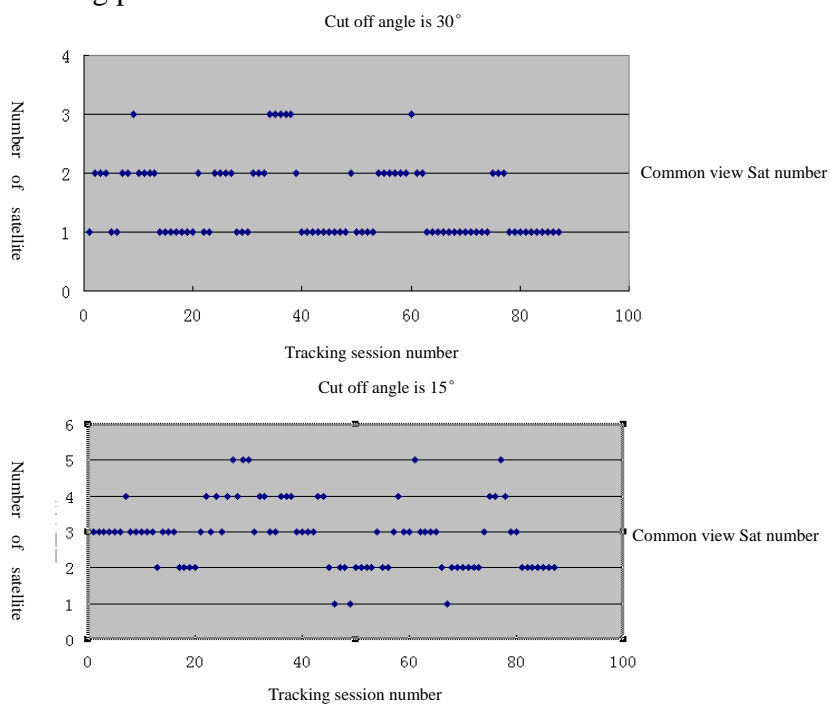

Figure 4: Number of GPS satellites that can be viewed by two 
stations at different cut-off altitudes during each track period

From the up of Figure 4, it can be seen that there are 2 satellites can be viewed at least together for each tracking period when the cut-off angle is $30^{\circ}$. From the lower of Figure 4 , we can see that there are at least $2 \sim 4$ at the elevation angle of $15^{\circ}$, and even a few tracking periods will even reach 5 common-view GPS satellites.

We calculated common-view data of the two-station with GGTTS standard format and elevation angle is selected to be $30^{\circ}$. there are at least two common-view satellites for each tracking period due to the multi-channel receiver be used. We have calculated the weighted average of all common-view GPS satellites in the same tracking period by using the weighted root mean square error of each satellite to obtain a time comparison between two stations. Figure 5 shows the change in the calculated synchronization frequency difference between the two stations:

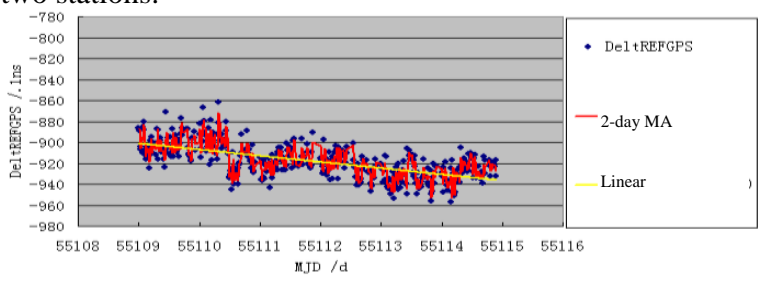

Figure 5: Relative changes in the comparison results between the PTB and USNO stations

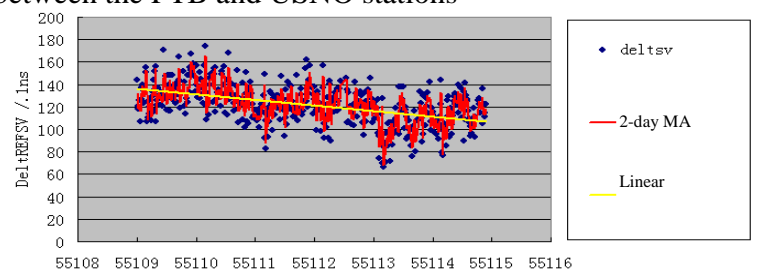

Figure 6: Relative changes in the synchronized value of the two-station frequency marker calculated by BIPM

As we can see from the above two graphs, our results have a same trend with the BIPM results, but there is a clear system difference between the two results, and the difference value can be clearly expressed in the following results. Our result is compared with BIPM's consensus results. The difference (without taking into account hardware delay corrections) are shown in Figure 7:

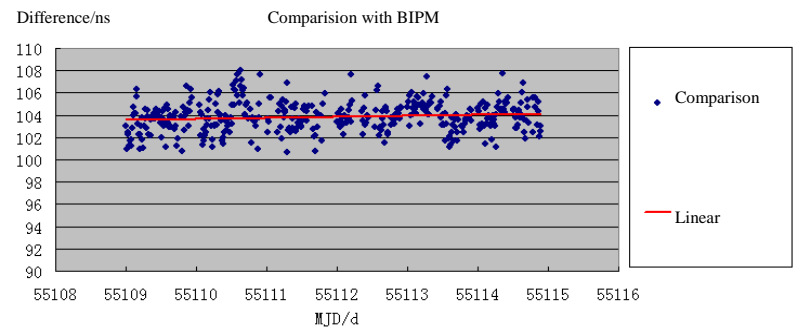

Figure 7: Differences between the results of this paper and BIPM

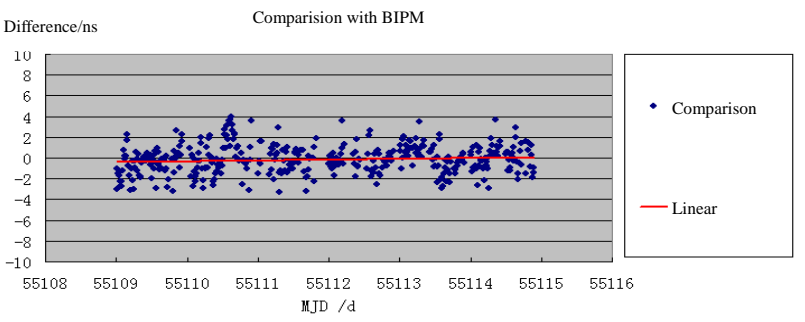

Figure 8: Result of comparison with other factors such as hardware delay.

\section{CONCLUSION}

This paper processed six days data of station PTB and station USNO. It can be seen from the root mean square error of the REFSV shows a decreasing trend within a week long time, It's tends to be stable. The mean square error shows a linear change, but jump occurs across days. It is estimated that the jumps caused by satellite precise ephemeris is used for interpolation when performing satellite position interpolation. Compared our results with the results obtained by BIPM, we can see that the difference is among $102 \mathrm{~ns}$ to $106 \mathrm{~ns}$ which regardless of the hardware delay. The difference is mostly lower than $2 \mathrm{~ns}$ when we taking into account the systematic bias mainly caused by hardware delay, but occasional differences almost reach $4 \mathrm{~ns}$ in individual epoch. The data was processed by using Black Troposphere Delay model, It may be the troposphere delay correction is not completely cleared since the distance between two stations is too long (about $6000 \mathrm{~km}$ ), and the multi-path effect at each station is also must be considered. For a week-long observation data, the hardware delay is considered to be constant when we processing data, Actually, the hardware delay has slight change within a day and may have a great change within a week which affected by the temperature .The hardware delay must be considered in high accuracy data processing.

\section{ACKNOWLEDGEMENTS}

The author would like to acknowledge the efforts of the IGS 2005) and BIMP for providing the GPS observation data and processing results. This work is supported by the National Natural Science Foundation of China (NO. 41504020) .

\section{REFERENCES}

1. Nie Guigen. Error Analysis and Application of High Accuracy GPS Time Measurement and Time Transfer,[D],2002, WuHan University Press, 114-124

2. Zhou Zhongmo. Principle and Application of GPS. Beijing. Surveying and Mapping Press.1992.

3. Li Zhenghang GPS Surveying and Processing. Wuhan, Wuhan University Press,2005.

4. http://www.BIPM.org / www.igscb.jpl.nasa.gov

5. Wu Di .GPS Common View Method for Remote Time and Frequency Transfer.[2007],33-34

6. Xu QiFeng, GPS Navigation and Positioning .BEIJING PLA Publishing Press 1994.

7. Guigen Nie,Kefei Zhang,Falin Wu,Bo Zhu, Research on LEO Satellites Time Synchronization with GPS Receivers Onboard, Proceedings of the Joint 2007 European Frequency and Time Forum \& 2007 IEEE Frequency Control Symposium 896-900

8. D.W.Allan and C.Thomas. Technical Directives for Standardization of GPS Time Receiver Software to be implemented for improving the accuracy of GPS common-view time transfer. Metrologia ,[1994],69-79

9. M.A.Weiss. A comparison of GPS Common-View Time Transfer to All-in-View. Time and Frequency Division NIST.[2005] 324-328

\section{Revised March 2018}

